\title{
Robust offset locking of laser frequency with electronically tunable LC circuits for sub-millihertz uncertainty
}

\author{
Yuhei Seishu ${ }^{1} \cdot$ Taro Hasegawa $^{1,2}$
}

Received: 11 April 2019 / Accepted: 2 July 2019 / Published online: 12 July 2019

(c) The Author(s) 2019

\begin{abstract}
A simple scheme for laser frequency offset locking introduced in Cheng et al. (Opt Express 25:2752, 2017) has been improved by the use of a variable-capacitance diode. By employing this scheme with the optical phase lock loop, robust and precise laser frequency stabilization is achieved, and long-term laser frequency drift is expected to be substantially suppressed even in noisy circumstances. The optical frequency uncertainty becomes less than $10 \mu \mathrm{Hz}$ for $1000 \mathrm{~s}$ averaging.
\end{abstract}

\section{Introduction}

Laser frequency stabilization is one of the essential technique in atomic, molecular, and optical physics, quantum optics, optical communications, and fundamental physics [1, 2]. Optical offset locking is the technique to stabilize optical frequency of a single-mode laser (slave laser) with respect to another well-stabilized laser (master laser) by means of beat-note frequency stabilization. Especially, after the invention of optical frequency combs (OFC), the optical offset locking technique is indispensable to these applications to obtain highly frequency-stabilized coherent light sources of the wavelength range from ultraviolet to mid-infrared [3]. By optical phase lock loop (OPLL) [4], which is one of the offset locking schemes, the slave laser frequency can be stabilized with very small uncertainty (less than millihertz). The OPLL is also employed to stabilize carrier-envelope offset frequency of OFCs [5]. With the OPLL, however, large optical frequency noise cannot be suppressed. If the phase difference between the master laser and the slave laser exceeds $\pi$ within response time of the feedback loop, the OPLL does not work any more. Hence, the OPLL cannot be employed in noisy circumstances such as rooms with substantial air flow and acoustic noises, and the outside of laboratories [6]. Because of this reason, another stabilization

Taro Hasegawa

hasegawa@phys.keio.ac.jp

1 Department of Physics, Keio University, 3-14-1, Hiyoshi, Kohoku, Yokohama, Kanagawa 223-8522, Japan

2 JST, ERATO, MINOSHIMA Intelligent Optical Synthesizer Project, Tokyo, Japan scheme in addition to the OPLL is necessary to suppress frequency jumps (jitters). For this purpose, digital processing technique [7], optical frequency locking to resonance frequency of a high-finesse cavity [8], offset locking with an electric delay line $[9,10]$, and offset locking with a low-pass filter [11], and offset locking with an LC resonance circuit (LC locking) [12] are utilized. The LC locking is a scheme in which the beat-note frequency between the master and the slave lasers is stabilized to the LC resonant frequency. This scheme is simple compared to that using a high-finesse cavity, and it is easily used with the OPLL. When the LC locking and the OPLL are employed simultaneously, robust and precise offset locking is realized. Robustness of the frequency locking is supported by the LC locking, whereas small uncertainty is supported by the OPLL, as in [12]. In addition, the offset frequency in the LC locking is manually tunable if the capacitance is variable (as in Fig. 1 in [12]), whereas tuning range of the electric delay line is very small. Therefore, the LC locking is very useful, especially in noisy circumstances, with no digitizing noise, which may appear in the digital processing technique [7].

When the LC locking is employed with the OPLL, the offset frequency of the OPLL [frequency of the local oscillator (LO)] is supposed to be identical to the locking offset frequency of the LC locking (the LC resonance frequency). The mismatch between these two frequencies causes the phase shift of the slave laser with respect to the master laser. As a result, the LC resonance frequency fluctuation causes the phase noise, and long-term stability of the laser frequency deteriorates.

To overcome this problem, we improve the LC locking introduced in [12] by making the resonance frequency 
tunable electronically. Specifically, we replace the manually variable capacitance in Fig. 1 in [12] with a variable capacitance diode (varactor). With an electronically tunable LC circuit, electronic tuning of the beat-note frequency can be carried out. The LC resonance frequency can be electronically stabilized to the LO frequency in the OPLL, and with this stabilization, long-term fluctuation of the slave laser frequency is suppressed. As a result, the electronically tunable LC locking can improve long-term laser frequency stability with maintaining the robustness.

In our experiment, an external-cavity laser diode (ECLD) at $1645 \mathrm{~nm}$ is the slave laser, and an OFC of erbium-doped fiber mode-locked laser is the master laser. We demonstrate the LC locking in addition to the OPLL, and the uncertainty of the beat-note frequency is the order of $10 \mu \mathrm{Hz}$ for $1000 \mathrm{~s}$ of measurement averaging time. The behavior of the overlapping Allan deviation indicates that the long-term drift of the beat-note frequency is substantially suppressed even in noisy circumstances in our laboratory. From this result, it is found that the deterioration of OPLL performance in exchange for the robustness of LC locking can be avoided by the use of the electronically tunable LC locking.

\section{Principles and experimental setup}

\subsection{Tunable LC circuits}

As in [12], the beat-note frequency of two lasers is to be stabilized at the resonance frequency of an LC circuit. In our setup, we use a varactor to tune the LC resonance frequency. The circuit is shown in Fig. 1. The value of the inductance is $1 \mu \mathrm{H}, \mathrm{C} 1$ is $10 \mathrm{nF}, \mathrm{C} 2$ is $1 \mathrm{nF}$, and $\mathrm{R}$ is $1 \mathrm{k} \Omega$. For the varactor (VC), we use 1SV228 (Toshiba). The capacitance of the varactor is about $45 \mathrm{pF}$ when $0 \mathrm{~V}$ is applied as a control voltage $\left(V_{\mathrm{Ctrl}}\right.$ in Fig. 1), and about $10 \mathrm{pF}$ for $9 \mathrm{~V}$. The condenser $\mathrm{C} 1$ is necessary to apply a dc voltage to the varactor. Because the capacitance of $\mathrm{C} 1$ is much larger than that of the varactor, the resonance frequency of the LC circuit $\omega_{0} / 2 \pi$ is determined by the capacitance of the varactor. The resonance frequency is expected to be tunable between 24 and 50 MHz. Practically, however, because of stray capacitance and inductance, the resonance frequency turns out to be tunable between 16 and $40 \mathrm{MHz}$ as shown later.

The rf power $\left(P_{\text {in }}\right)$ is injected through the rf terminal ( $\mathrm{rf}$ in Fig. 1), and the reflected $\mathrm{rf}$ power $\left(P_{\text {ref }}\right)$ is utilized to obtain the error signal for the laser frequency offset locking. Figure 2 shows the rf-frequency dependence of (a) the rf reflection amplitude and (b) the out-of-phase component (the component of quadrature phase with respect to the input rf signal) for some specific values of $V_{\mathrm{Ctrl}}$. The stray resistance in the circuit determines the linewidth of the resonance curve. When the control voltage is $\sim 3.00 \mathrm{~V}$, the reflection

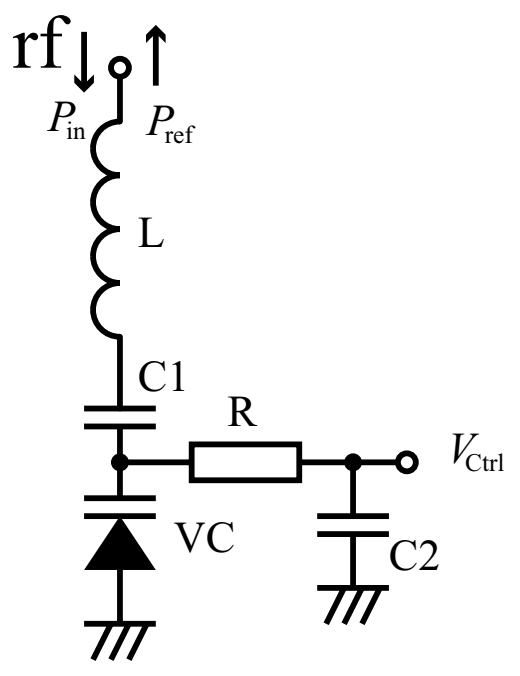

Fig. 1 The LC resonator with electronically tunable resonance frequency. $\mathrm{L}$ is the inductance, $\mathrm{C} 1$ and $\mathrm{C} 2$ are the condensers, $\mathrm{R}$ is the resistance, and $\mathrm{VC}$ is the varactor. The terminal $V_{\mathrm{Ctrl}}$ is for capacitance control of $\mathrm{VC}$, and $\mathrm{rf}$ power is injected and reflected back through the rf terminal

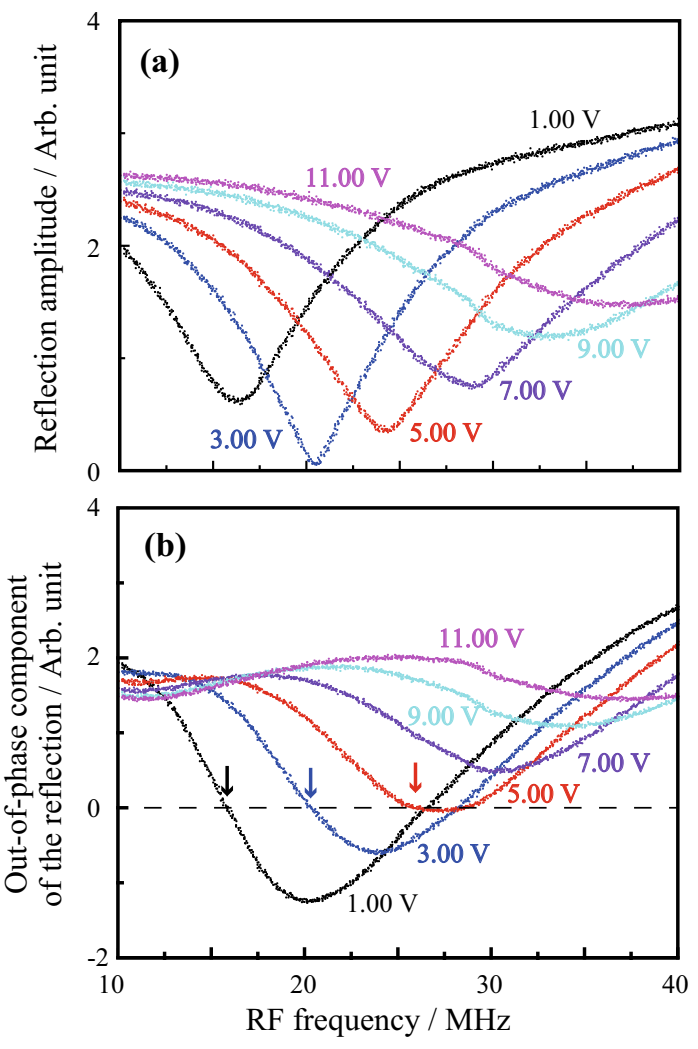

Fig. 2 Dependence of $\mathbf{a}$ the reflection amplitude and $\mathbf{b}$ out-of-phase component on the input rf frequency. The control voltage is shown in the figure. The arrows in $\mathbf{b}$ indicate the zero-cross points, at which the beat-note frequency is to be stabilized 
amplitude is almost zero level at the resonance ( $\sim 20 \mathrm{MHz})$, so that the impedance matching condition is fulfilled. The out-of-phase component is used as an error signal for the laser frequency offset locking, and the beat-note frequency is to be stabilized at zero-cross frequency in Fig. $2 b$ (arrows in the figure). Because of the background profile, the zero-cross frequency appears only when the control voltage is less than $5 \mathrm{~V}$ (between 16 and $25 \mathrm{MHz}$ ). In the case of impedance matching, the reflection power consists of only the out-ofphase error signal in the vicinity of the resonance frequency, and irrelevant on-phase component, which may not contribute to the signal but cause noise, is negligibly small. With the present circuit, it is practical to tune the resonance frequency to $\sim 20 \mathrm{MHz}$ for the LC locking. In Fig. $2 \mathrm{~b}$, there is another zero-cross frequency (e.g., $\sim 28 \mathrm{MHz}$ for $V_{\text {Ctrl }}=3.0$ $\mathrm{V})$, and locking to this frequency can be avoided by choosing the feedback polarity properly.

One of the important parameters of the tunable LC circuits is tuning range of the resonance frequency, because it determines the tuning range of the slave laser frequency stabilized by the LC locking. The tuning range $\left(\Delta f_{\mathrm{LC}}\right)$ is determined by the variable range of the capacitance, and it is expressed as,

$2 \pi \Delta f_{\mathrm{LC}}=\frac{1}{\sqrt{\mathrm{LC}_{\min }}}-\frac{1}{\sqrt{\mathrm{LC}_{\max }}}$,

where $L$ is the inductance, and $C_{\min , \max }$ are the smallest and the largest capacitance of the varactor, respectively. Equation (1) implies that smaller value of $L$ (or higher resonance frequency) is preferred for wide tuning range. In our case, the beat-note frequency is $\sim 20 \mathrm{MHz}$, which is limited by the repetition rate (or mode spacing in frequency domain) of the OFC ( $66.87 \mathrm{MHz})$.

Another important parameter is capture range, within which sudden laser frequency error can be compensated by the feedback loop. For $V_{\mathrm{Ctrl}}=3 \mathrm{~V}$, the capture range is roughly estimated as $\sim 10 \mathrm{MHz}$ (frequency range between the maximum and the minimum of the error signal). This capture range is much larger than that of the OPLL (estimated later as $150 \mathrm{kHz}$ ), implying the robustness of the $\mathrm{LC}$ locking.

\subsection{Lasers}

In this study, optical frequency of an ECLD with the grating feedback at $1645.8 \mathrm{~nm}$ (slave laser) is offset-locked with respect to one mode frequency of an OFC (master laser). These lasers have been used in another study [13, 14]. Briefly, the OFC is a mode-locked erbium-doped fiber laser with a repetition rate of $66.87 \mathrm{MHz}$, whose bandwidth is broadened by the use of an optical amplifier and a highlynonlinear optical fiber to cover the ECLD wavelength.
The linewidth of each mode of the OFC is less than 1 $\mathrm{Hz}$, whereas that of the ECLD is over $200 \mathrm{kHz}$ without stabilization.

\subsection{OPLL with LC locking}

Linewidth of the beat-note spectral line with the LC locking is expected to be a few kilohertz [12], and for narrower linewidth down to the order of sub-millihertz, OPLL is carried out in addition to the LC locking. When the two locking schemes are employed together, narrow linewidth (by the OPLL) and robustness against the jitters (by the LC locking) are simultaneously attained.

For simultaneous use of the LC locking and the OPLL, the two error signals have been simply added in [12]. The OPLL is for stabilizing the beat note phase, and hence the locked beat-note frequency may be determined only by the LO frequency for the OPLL. Then difference of the LC resonance frequency from the $\mathrm{LO}$ frequency seems not to cause frequency shift at all, and instead, it causes only phase shift, as experimentally demonstrated below. However, fluctuation of the LC resonance frequency induces the stabilized beatnote frequency fluctuation, as it is derivative of the phase by time. Such fluctuation of the LC resonant frequency may be due to temperature drift and gives rise to slow beat-note frequency fluctuation (rough estimation is given in Sect. 3). Consequently, when employing the LC locking for the robustness without sacrificing the long-term stability of the OPLL, it is important to make the LC resonant frequency identical with the LO frequency.

In general, the OPLL is the best scheme to precisely lock the beat-note frequency and, therefore, it is favored to fix the $\mathrm{LO}$ frequency and to tune the $\mathrm{LC}$ resonant frequency to the LO frequency. Then, in this study, the tunability of the LO frequency is reserved for other purpose, such as tuning or scanning the ECLD optical frequency with fixed OFC frequency, and the LC resonant frequency is to be tuned at the $\mathrm{LO}$ frequency.

\subsection{Overall experimental setup}

The overall experimental setup for the optical frequency stabilization is schematically shown in Fig. 3. The beat-note signal between the OFC and the ECLD is detected by balanced photodetectors to eliminate irrelevant signal at the repetition rate of the OFC and its harmonics $[13,14]$. The beat-note detection is carried out by two sets of the balanced detection. One is for the laser frequency stabilization described later, and the other is for monitoring the beatnote signal. For convenience, the beat-note signal for the ECLD frequency stabilization is referred as "in-loop signal", and that for beat-note monitoring is as "out-of-loop signal" hereafter. 


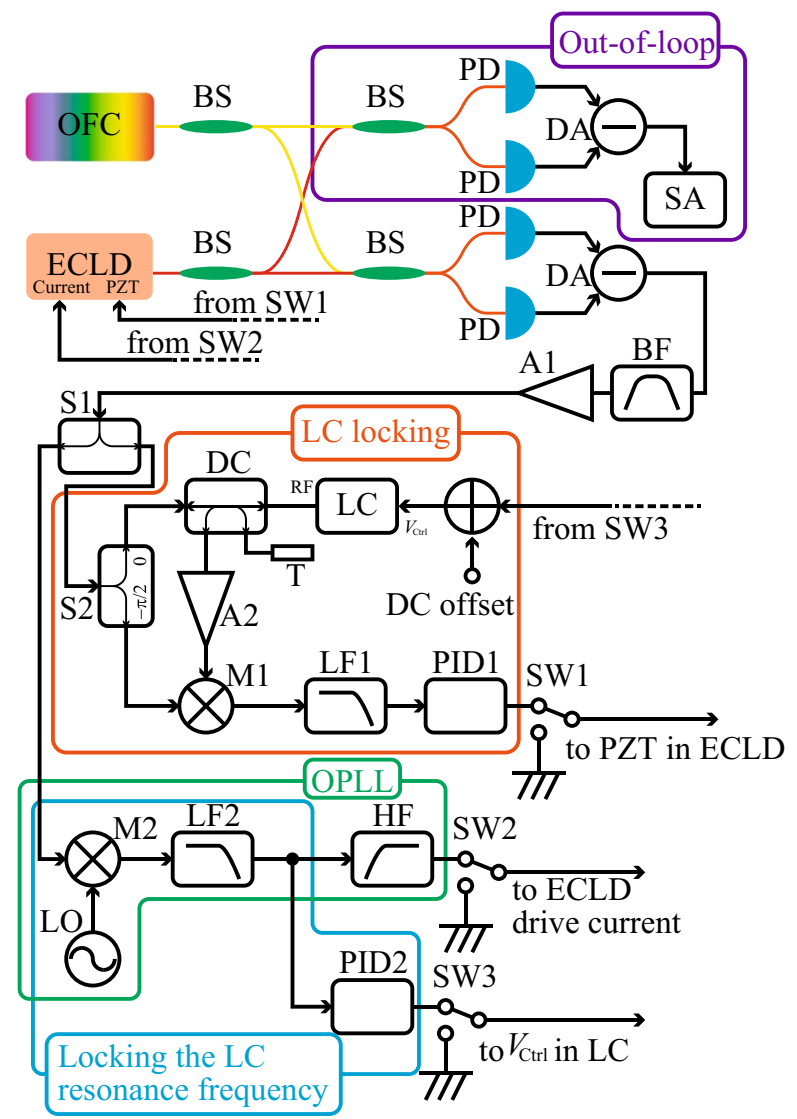

Fig. 3 Experimental setup. Abbreviations not mentioned in the text: BS for 50:50 optical beam splitters, PD for photodetectors, DA for differential amplifiers, T for a $50 \Omega$ terminator, LF1 and LF2 for lowpass filters (cut-off frequency is $1.9 \mathrm{MHz}$ for LF1 and $5 \mathrm{MHz}$ for LF2), and HF for a high-pass filter

As stated before, the ECLD frequency is to be stabilized with respect to the OFC mode frequency. To be exact, what is stabilized is the ECLD frequency at the photodetectors for the in-loop signal rather than the frequency at the output of the ECLD device. When the ECLD frequency linewidth is broad (over $100 \mathrm{~Hz}$ ), the ECLD frequency at the photodetectors can be considered as that at the output of the ECLD device. However, for narrow linewidth (less than $100 \mathrm{~Hz}$ ), these two ECLD frequencies are different each other because of phase noise induced by mechanical fluctuation on optical fibers. In the experimental setup, optical path lengths of in-loop and out-of-loop fibers for the OFC are $\sim 3 \mathrm{~m}$ and $\sim 4 \mathrm{~m}$, respectively, and each of those for the ECLD is $\sim 1$ $\mathrm{m}$. These fibers mechanically fluctuate because of air flow from air conditioning. The out-of-loop beat-note frequency spectrum observed by the rf spectrum analyzer is with the fiber noise, whereas the in-loop signal is without the fiber noise if the feedback loop works properly. Hence stability of the in-loop signal frequency is the measure of the feedback performance. It should be noted that, with the stabilization, the phase noise caused by the in-loop fiber is on the output immediately after the ECLD device, as the feedback loop works to cancel the phase noise at the in-loop photodetectors. As a result, the out-of-loop noise consists of the phase noises from both of the out-of-loop fiber and the in-loop fiber.

The out-of-loop beat-note signal is monitored by a rf spectrum analyzer (SA, FSC3 of Rohde and Schwarz) as shown in the frame named "out-of-loop" in Fig. 3. The in-loop beat-note signal detected by balanced detectors (PDB480C-AC of Thorlabs) passes through a bandpass filter (BF, 19.2-23.6 MHz, SBP-21.4+ of Mini-Circuits) and a rf amplifier (A1, $15 \mathrm{~dB}, \mathrm{AA} 170$ of R and $\mathrm{K}$ ). The bandpass filter is necessary to eliminate spectral components except at the lowest beat-note frequency (note that the beat note consists of frequency components at $N f_{\text {rep }} \pm \delta f$, where $N$ is an integer, $f_{\text {rep }}$ is the repetition rate of the OFC, and $\delta f$ is the lowest beat-note frequency). The beat-note signal is split into two parts by a power splitter (S1, ZFSCJ-2-1-S+ of Mini-Circuits). One is for the tunable LC locking, and the other is for the OPLL.

The beat-note signal for the LC locking is further divided into two parts by the power splitter with $\pi / 2$ phase shift (S2, QH040-0S of R and K). One is injected into the tunable LC circuit of Fig. 1 (LC in Fig. 3) through the directional coupler (DC, ZFBDC20-61HP+ of Mini-Circuits). The rf signal from the reflection port of the DC [amplified by an amplifier (A2, $40 \mathrm{~dB}, 493 \mathrm{~A} / 40$ of Perry Amplifier)] and the phase-shifted rf signal of S2 are mixed (M1, ZAD3+ of Mini-Circuits) to detect the out-of-phase component of the reflection power from the tunable LC circuit. The low-frequency component of the output from the mixer is the error signal for stabilizing the beat-note frequency at the LC resonance. After adjusted by a handmade proportional-integralderivative (PID) controller, it is fed back to the piezoelectric transducer (PZT) in the ECLD, with which the laser cavity length can be adjusted. The response time of the PZT determines the feedback bandwidth of the LC locking $(\sim 10 \mathrm{kHz})$. The feedback loop can be closed with a switch (SW1).

For the OPLL, the other output of S1 is mixed by a mixer (M2, MX010-0S of R and K) with a LO signal (AFG3022C of Tektronix), and after a low-pass filter (LF2, $5 \mathrm{MHz}$ ) and a handmade high-pass filter (cut-off frequency is $120 \mathrm{~Hz}$ ), the error signal component faster than $120 \mathrm{~Hz}$ is fed back to the driving current of the ECLD after amplitude adjustment (not shown in the figure) for optimizing the OPLL. A switch (SW2) is used to close the OPLL as well.

To tune the LC resonant frequency, the OPLL signal is fed back to the control voltage of the tunable LC circuit. As shown in Fig. 3, after adjusted at another PID controller (PID2) and added to an offset dc voltage, the OPLL signal is fed back to the varactor control voltage. A switch (SW3) is used to close this feedback loop. 


\section{Results and discussions}

First, we demonstrate only the LC locking (SW1 is closed, and SW2 and SW3 are grounded). The out-of-loop rf spectrum of the beat-note signal is shown in Fig. 4. The control voltage is given in the figure. The full-width-at-half-maximum linewidth is $24 \mathrm{kHz}$, which is worse than the result in [12] (2 kHz). The linewidth is determined by the signalto-noise ratio of the beat-note signal and slope of the error signal at the lock point shown in Fig. 2. In our case, the mode intensity of the master laser (the OFC, average power in a single mode is $100 \mathrm{nW} /$ mode [13]) is anticipated to be weaker than the continuous-wave laser in [12], resulting smaller signal-to-noise ratio of the beat-note signal. If the slope of the error signal in this study is not so different from that in [12], the wider linewidth may be due to smaller signal-to-noise ratio.

The offset frequency can be changed by tuning the control voltage, as shown in Fig. 4. The tuning range of the offset frequency is between 17.9 and $22.1 \mathrm{MHz}$ for $2.0 \mathrm{~V}<V_{\mathrm{Ctrl}}<$ $4.0 \mathrm{~V}(2.1 \mathrm{MHz} / \mathrm{V})$. This result seems that the tuning range is so narrow $(\sim 4 \mathrm{MHz})$. This range is limited by the bandwidth of the bandpass filter (BF in Fig. 3). As described before, the bandpass filter is required to eliminate irrelevant rf spectral components, and hence essentially the tuning range is determined by the repetition rate (mode spacing) of the OFC. The combination of a high-repetition-rate OFC and an LC circuit of high resonant frequency is expected to provide wide tuning range of the beat-note frequency.

Next, the OPLL is closed in addition to the LC locking (SW1 and SW2 are closed, and SW3 is grounded). This measurement is carried out to observe effects of the frequency mismatch between the LO frequency for the OPLL and the LC resonant frequency for the LC locking. For this

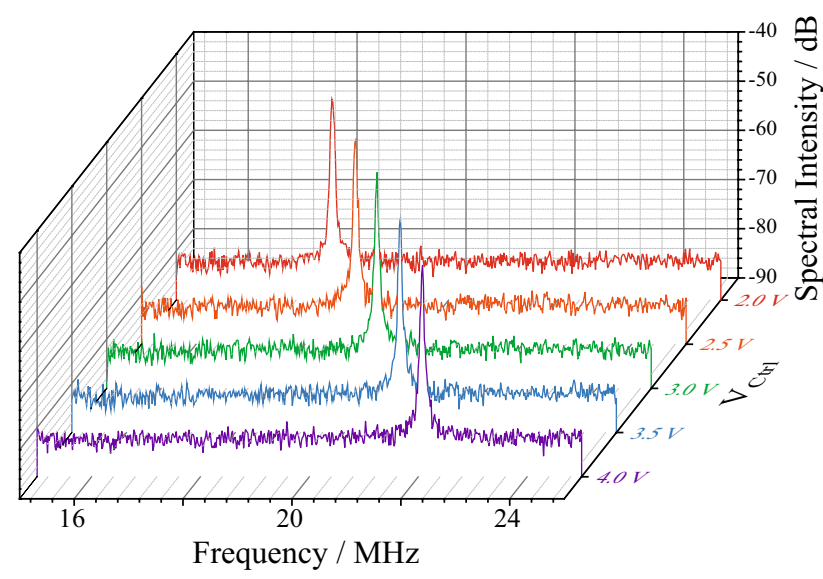

Fig. 4 Stabilized out-of-loop beat-note spectra with the tunable LC offset locking for some specific values of the control voltage. The resolution bandwidth of the spectrum analyzer is $10 \mathrm{kHz}$ purpose, components slower than $120 \mathrm{~Hz}$ in the OPLL error signal are not fed back to the ECLD (the slow feedback is only by the LC locking). Then we expect that we can estimate how much the optical phase stabilized by the OPLL is shifted by the frequency mismatch by monitoring slow (dc) component of the OPLL error signal.

The LO frequency is set at $20 \mathrm{MHz}$. When the control voltage of the LC circuit is $3.012 \mathrm{~V}$ and $3.034 \mathrm{~V}$, behavior of the error signal of the OPLL (output of the LF2 in Fig. 3) indicates that mode hopping of the laser frequency occurs repeatedly (Fig. 5a, d, respectively). When the mode hopping occurs, the phase difference between the beat note and the LO changes by $2 \pi$, and the transient time of the mode hopping is the order of the OPLL feedback time constant (10 $\mu \mathrm{s})$. This phase change can be seen as a pulse-like behavior in Fig. 5a, d. Even with the mode hopping, the slave laser frequency is still stabilized owing to the robustness of the LC locking. When $V_{\text {Ctrl }}$ is $3.020 \mathrm{~V}$ and $3.028 \mathrm{~V}$ (Fig. 5b, c, respectively), no mode hopping occurs, but the beat-note phase is not stabilized to zero. In these cases, the beat-note frequency is locked to the LO frequency, since the phase is almost constant. However, if the LC resonant frequency drifts (by temperature drift, for example), the phase drift induces beat-note frequency shift as well. In addition, in the cases of Fig. 5b, c, the mode hopping occurs after several minutes of stabilization. We consider this mode hopping is indeed induced by the LC resonant frequency drift. Even if the feedback signal is the sum of the OPLL error signal and the LC locking error signal as in [12], the beat-note frequency drift appears, because the feedback loop works so that the two error signals cancel each other.

From the result in Fig. 5, we can roughly estimate how much the frequency drift is anticipated when the sum of the OPLL error signal and the LC locking error signal is fed back to the ECLD as in [12]. The result in Fig. 5 implies that the phase shift of the beat-note signal is roughly $\pi$ when $V_{\mathrm{Ctrl}}$ is modified by $0.01 \mathrm{~V}$. From the result in Fig. 4, the change in $V_{\text {Ctrl }}$ by $0.01 \mathrm{~V}$ corresponds to the $\mathrm{LC}$ resonant frequency shift of $21 \mathrm{kHz}$. Therefore, taking $20 \mathrm{MHz}$ of the LC resonant frequency into account, drift of the LC resonant frequency by a factor of $10^{-3}$ corresponds to the phase shift of $\pi$. To achieve $1 \mathrm{mHz}$ uncertainty of the beat-note frequency, the stability of the LC resonant frequency should be better than $2 \times 10^{-3}$ in $1000 \mathrm{~s}$. As typical stability of capacitance in LC circuits is about $0.2 \%$ for temperature change of 1 ${ }^{\circ} \mathrm{C}$ [15], temperature of the LC circuit should be stabilized within the range of $1{ }^{\circ} \mathrm{C}$ for $1000 \mathrm{~s}$ (temperature dependence of inductance should be taken into account as well).

When the $\mathrm{LC}$ resonant frequency is stabilized to the $\mathrm{LO}$ frequency, then we do not have to make an effort to eliminate the temperature drift and other noises, and it is expected that long-term stability of the beat-note frequency is improved. For this purpose, the slow component (less than $1 \mathrm{kHz}$ ) of 


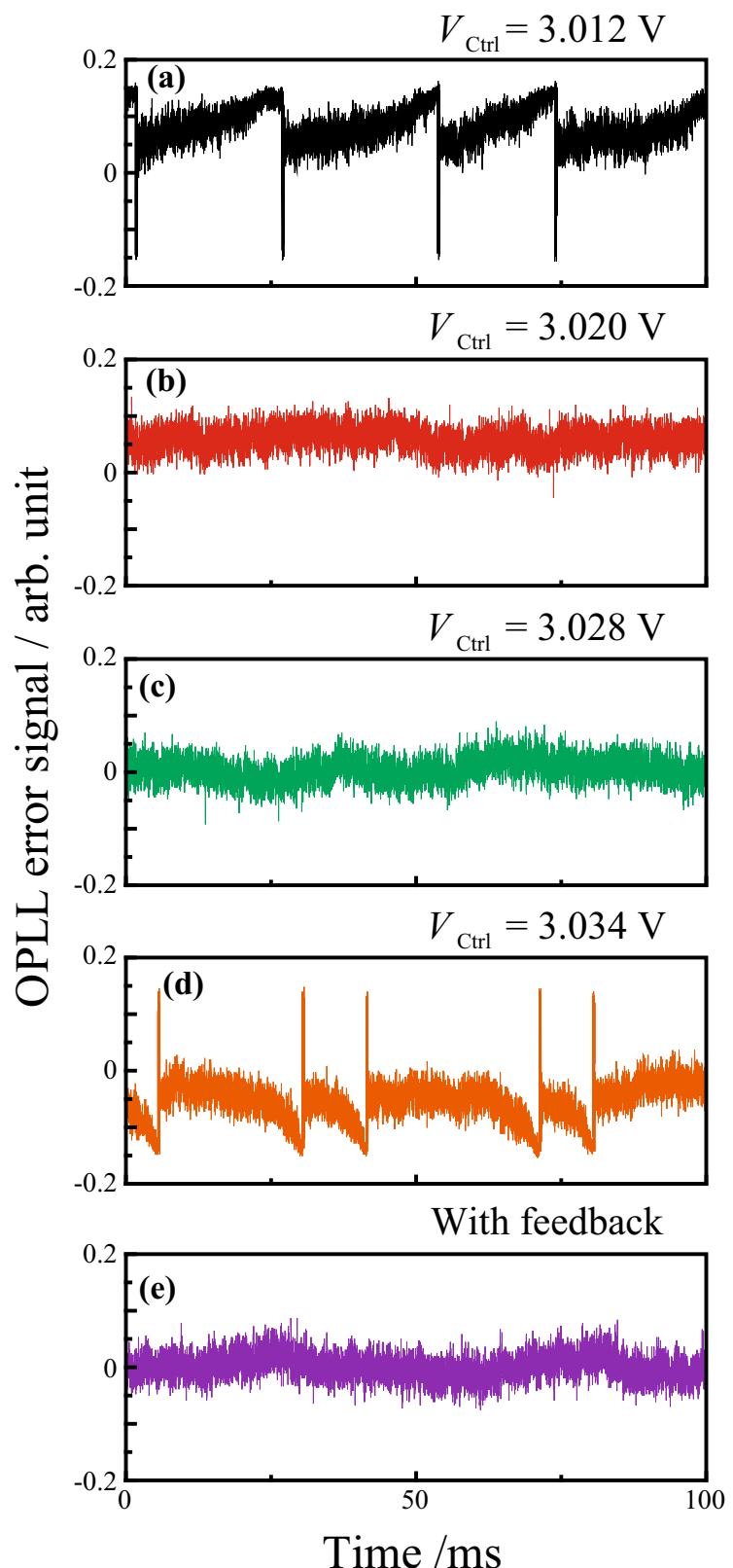

Fig. 5 The OPLL error signal when the OPLL is closed for component faster than $120 \mathrm{~Hz}$ of the error signal (SW1 and SW2 are closed and SW3 is grounded). In a-d, the control voltage for the LC circuit is set at the value shown in each figure. In e, the OPLL error signal is fed back to the control voltage of the LC circuit (SW3 is closed) to make the LC resonant frequency identical to the LO frequency. The sampling rate is $10 \mu \mathrm{s}$

the OPLL feedback signal is fed back to the control voltage of the LC circuit (SW3 is closed). Then the LC resonant frequency is automatically tuned to the LO frequency, and the OPLL error signal is stabilized at zero (Fig. 5e, which corresponds to the phase noise in the locked condition). The beatnote frequency is well locked, and its spectrum is shown in Fig. 6. The feedback bandwidth for the OPLL turns out to be

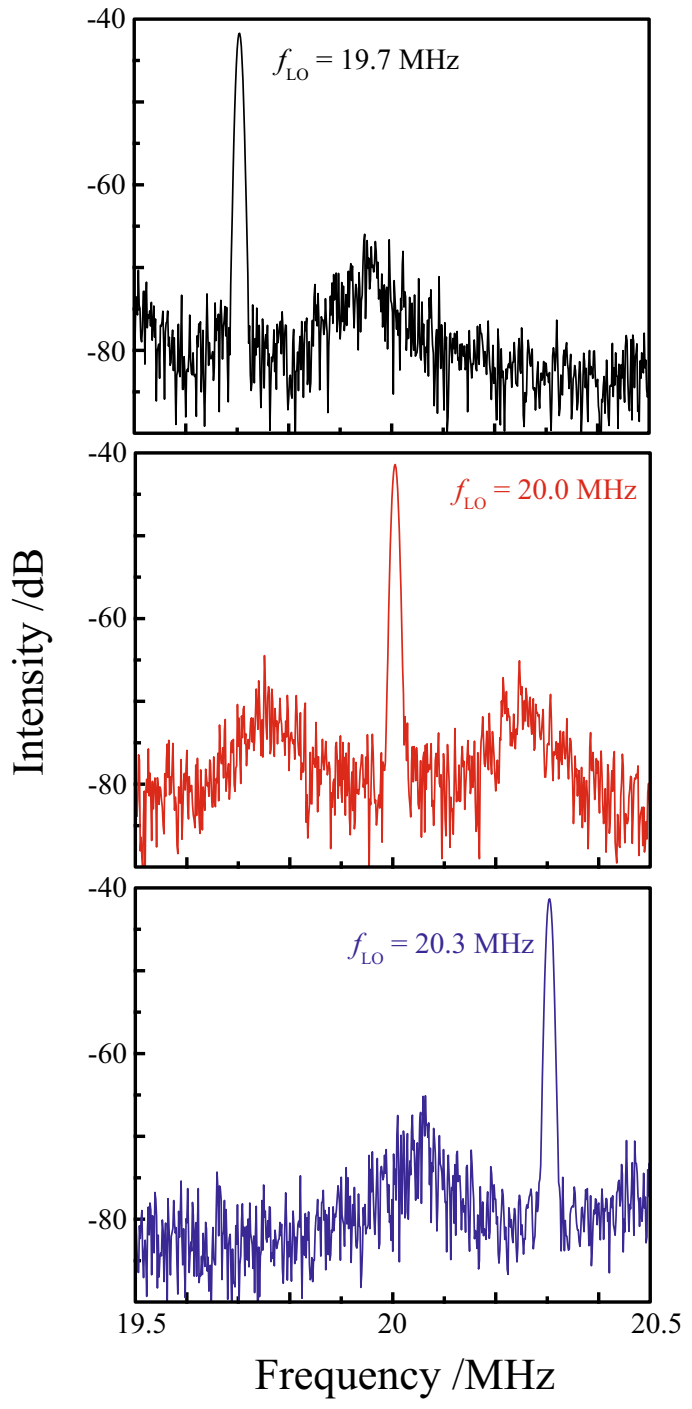

Fig. 6 The out-of-loop beat-note rf spectra with the resonant frequency stabilization to the $\mathrm{LO}$ frequency. The LO frequency $f_{\mathrm{LO}}$ is given in the figure. The resolution bandwidth of the rf spectrum analyzer is $1 \mathrm{kHz}$, which determines the spectral linewidth in the figure

$150 \mathrm{kHz}$, which is estimated from the sidebands that appear in Fig. 6 (servo bumps). The feedback bandwidth is smaller than the linewidth of the free-running ECLD $(200 \mathrm{kHz})$ and, therefore, the laser frequency stabilization only by the OPLL fails so often (once in a few seconds). By employing the LC locking with the OPLL, the feedback loop works for a long period of time (more than an hour). When the LO frequency is set from $20.0 \mathrm{MHz}$ (Fig. 6b) to $19.7 \mathrm{MHz}$ or $20.3 \mathrm{MHz}$, the slave laser frequency is kept stabilized without manual adjustment of $V_{\text {Ctrl }}$ (Fig. 6a, c).

To confirm the long-term stability, the overlapping Allan deviation of the beat-note frequency is estimated as shown in Fig. 7. The in-loop beat-note frequency is measured at the forward sampling port of the directional 


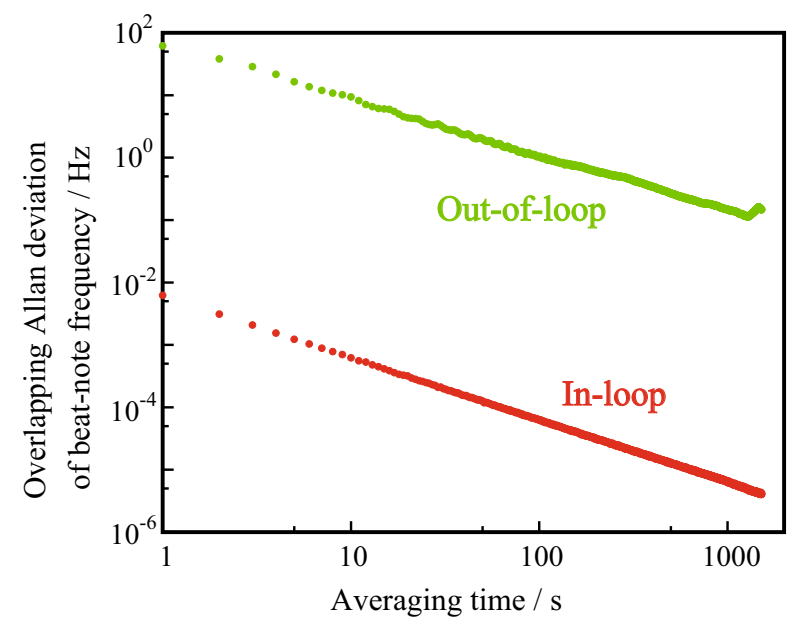

Fig. 7 The overlapping Allan deviation of in-loop and out-of-loop beat-note frequency

coupler by replacing the terminator $\mathrm{T}$ in Fig. 3 with a frequency counter (CNT-90 of Pendulum), and the outof-loop frequency is measured by replacing the spectrum analyzer with another frequency counter. The overlapping Allan deviation is estimated from 3000 successive measurements of the beat-note frequency by the frequency counter (each measured value is an average for $1 \mathrm{~s})$. The Allan deviation at $1 \mathrm{~s}$ averaging is $\sim 3 \mathrm{mHz}$, which is as low as that in [12]. The in-loop stability is still decreasing with inverse of the averaging time up to 1000 $\mathrm{s}$, resulting long-term drift of the beat-note frequency is suppressed. From this result, it is found that the use of an electronically-tunable LC circuit as the LC locking does not at all degrade the laser frequency uncertainty which is minimized by the OPLL, and robustness by the LC locking is attained. The high noise level of the out-ofloop frequency indicates that the present experiment has been carried out in very noisy circumstances. As stated before, the noise is mainly due to mechanical noise onto optical fibers. The mechanical noise is caused by air flow (in fact, air conditioning has been on during the measurement). Owing to robustness by the LC locking, the laser frequency stabilization works well even in such noisy circumstances. By the use of fiber noise cancellation technique [16], the out-of-loop noise can be reduced. The inloop Allan deviation with the $1000 \mathrm{~s}$ averaging is $\sim 10 \mu$ $\mathrm{Hz}$, and for longer averaging time it is expected to reduce further. The relative measurement uncertainty for $1000 \mathrm{~s}$ averaging is the order of $10^{-20}$ compared to the optical frequency. Therefore, this locking scheme does not degrade frequency stability when it is used with atomic clocks of the highest precision $[17,18]$.

\section{Conclusions}

In conclusion, we improve the robust laser frequency offset locking scheme using the LC resonance circuit introduced in [12]. By replacing the capacitance of the LC resonant circuit with a varactor, the $\mathrm{LC}$ resonant frequency becomes electronically tunable. When the OPLL and the LC locking are simultaneously employed, robust and precise locking can be performed. The robustness of the frequency locking is assured by the LC locking, and the preciseness is by the OPLL. By the electronic control, the LC resonant frequency can be stabilized to the local oscillator frequency used in the OPLL. With this stabilization, it is expected that the long-term phase noise caused by the LC resonant frequency drift is suppressed. In the demonstration, optical frequency of the ECLD is phase-locked with respect to a mode of the OFC. The uncertainty for $1000 \mathrm{~s}$-averaging is the order of $10 \mu \mathrm{Hz}$, and hence this scheme is applicable to experiments with uncertainty of the order of $10^{-20}$ even in noisy circumstances. The electronic tunability may be applicable to electric circuits utilized in other offset locking schemes such as that with low-pass filters [11].

Acknowledgements The authors acknowledge H. Sasada for discussions and comments. This study is financially supported by Japan Science and Technology Agency (JST) through the ERATO MINOSHIMA Intelligent Optical Synthesizer (IOS) Project.

Open Access This article is distributed under the terms of the Creative Commons Attribution 4.0 International License (http://creativeco mmons.org/licenses/by/4.0/), which permits unrestricted use, distribution, and reproduction in any medium, provided you give appropriate credit to the original author(s) and the source, provide a link to the Creative Commons license, and indicate if changes were made.

\section{References}

1. T. Udem, R. Holzwarth, T.W. Hänsch, Nature 416, 233 (2002)

2. J.P. Uzan, Rev. Mod. Phys. 75, 403 (2003)

3. K. Iwakuni, S. Okubo, O. Tadanaga, H. Inaba, A. Onae, F.-L. Hong, H. Sasada, Opt. Lett. 41, 3980 (2016)

4. A.M. Marino, C.R. Stroud, Rev. Sci. Instrum. 79, 013104 (2008)

5. H. Telle, G. Steinmeyer, A. Dunlop, J. Stenger, D. Sutter, U. Keller, Appl. Phys. B 69, 327 (1999)

6. J. Grotti, S. Koller, S. Vogt, S. Haefner, U. Sterr, C. Lisdat, H. Denker, C. Voigt, L. Timmen, A. Rolland, F.N. Baynes, H.S. Margolis, M. Zampaolo, P. Thoumany, M. Pizzocaro, B. Rauf, F. Bregolin, A. Tampellini, P. Barbieri, M. Zucco, G.A. Costanzo, C. Clivati, F. Levi, D. Calonico, Nat. Phys. 14, 437 (2018)

7. L. Cacciapuoti, M. de Angelis, M. Fattori, G. Lamporesi, T. Petelski, M. Prevedelli, J. Stuhler, G.M. Tino, Rev. Sci. Instrum. 76, 053111 (2005)

8. T. Day, E.K. Gustafson, R.L. Byer, IEEE J. Quantum Electron. 28, 1106 (1992)

9. U. Schünemann, H. Engler, R. Grimm, M. Weidemüller, M. Zielonkowski, Rev. Sci. Instrum. 70, 242 (1999) 
10. Y. Hisai, K. Ikeda, H. Sakagami, T. Horikiri, T. Kobayashi, K. Yoshii, F.-L. Hong, Appl. Opt. 57, 5628 (2018)

11. G. Ritt, G. Cennini, C. Geckeler, M. Weitz, Appl. Phys. B 79, 363 (2004)

12. W.-Y. Cheng, T.-J. Chen, C.-W. Lin, B.-W. Chen, Y.-P. Yang, H.Y. Hsu, Opt. Express 25, 2752 (2017)

13. T. Hasegawa, H. Sasada, Opt. Express 25, A680 (2017)

14. T. Hasegawa, Opt. Express 26, 24551 (2018)

15. Murata Manufacturing Co., Ltd., The temperature characteristics of electrostatic capacitance. https://www.murata.com/en-us/produ cts/emiconfun/capacitor/2012/10/15/en-20121015-p2. Accessed 10 July 2019
16. L.-S. Ma, P. Jungner, J. Ye, J.L. Hall, Opt. Lett. 19, 1777 (1994)

17. C.W. Chou, D.B. Hume, J.C.J. Koelemeij, D.J. Wineland, T. Rosenband, Phys. Rev. Lett. 104, 070802 (2010)

18. I. Ushijima, M. Takamoto, M. Das, T. Ohkubo, H. Katori, Nat. Photon. 9, 185 (2015)

Publisher's Note Springer Nature remains neutral with regard to jurisdictional claims in published maps and institutional affiliations. 